\title{
scONE-seq: A one-tube single-cell multi-omics method enables simultaneous dissection of molecular phenotype and genotype heterogeneity from frozen tumors
}

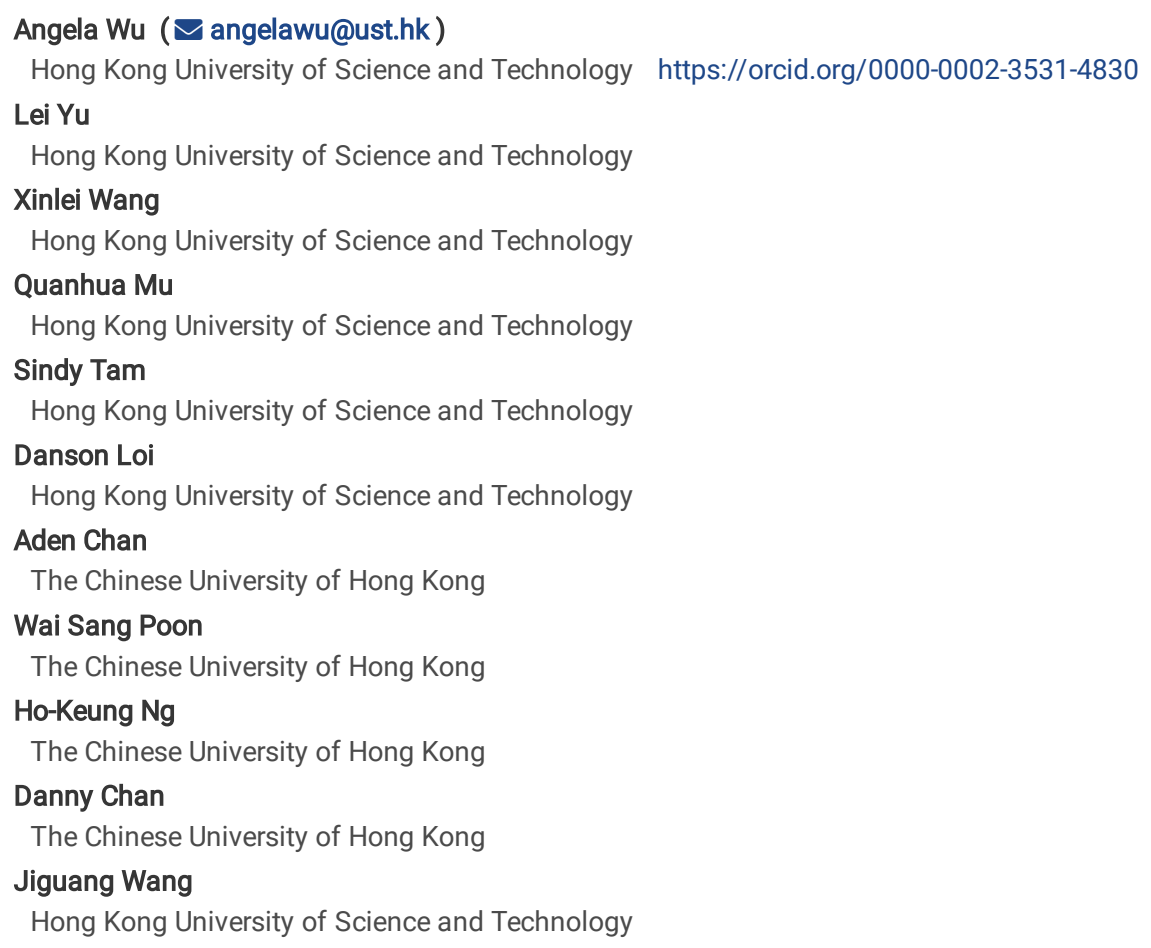




\section{Abstract}

Genomic and transcriptomic heterogeneity both play important roles in normal cellular function as well as in disease development. To be able to characterize these different forms of cellular heterogeneity in diverse sample types, we developed scONE-seq, which enables simultaneous transcriptome and genome profiling in a one-tube reaction. Previous single-cell-whole-genome-RNA-sequencing (scWGS-RNA-seq) methods require physical separation of DNA and RNA, often by physical separation of the nucleus from the cytoplasm. Most of these methods are labor-intensive and technically demanding, time-consuming, or require special devices, and they are not applicable to frozen samples that cannot generate intact single-cell suspensions. scONE-seq is a one-tube reaction which eliminates loss due to transfer steps, and thus is highly scalable and compatible with frozen biobanked tissue, generating data that is superior in quality compared to other applicable methods. We benchmarked scONE-seq against existing methods using cell lines and lymphocytes from a healthy donor, and we applied it to a 2-year-frozen astrocytoma sample profiling over 1,200 nuclei, subsequently identifying a unique transcriptionally normal-like tumor clone. scONE-seq makes it possible to perform large-scale single-cell multi-omics interrogation with ease on the vast quantities of biobanked tissue, which could transform the scale of future multi-omics single-cell cancer profiling studies.

\section{Introduction}

Single-cell genomics has become a mainstay technology used to dissect multicellular organisms and tissues that are composed of cells with diverse functions ${ }^{1-4}$. The power of this approach has been demonstrated in several cell atlas studies: novel cell types have been discovered that further led to the elucidation of new mechanisms; complex cellular interactions and transitions associated with disease initiation or progression have been revealed; crossspecies analyses have shed light on evolutionary processes ${ }^{5-8}$. The use of single-cell technology in studying cancer is especially important. Regulatory mechanisms underlying drug resistance or immune evasion are elusive and complex, and tumor cell heterogeneity is a major contributing factor to this complexity, making it particularly challenging to dissect these mechanisms with bulk techniques ${ }^{9-11}$. Single-cell technologies have greatly enhanced our understanding of tumor heterogeneity and accelerated mechanistic discovery. At the phenotype level, single-cell RNA-seq (scRNA-seq) has been used to uncover drug-resistant melanoma subpopulations and to characterize cancer stem cell subpopulations in glioblastoma ${ }^{7,12-15}$. scRNA-seq has also enabled a more comprehensive phenotypic understanding of the tumor microenvironment (TME) in many cancers including glioma and colorectal cancer ${ }^{16-20}$. At the genotype level, genomic instability contributes to cancer initiation, progression, relapse, and metastasis ${ }^{9}$. With single-cell whole genome sequencing (scWGS), the clonal structure of the tumor can be resolved, and evolutionary analysis based on copy number aberrations (CNAs) can reveal tumor progression ${ }^{21,22}$.

Evidently, both the genomic and transcriptomic heterogeneity of tumors contributes to the disease, and understanding the importance of both in cancer studies is crucial. Several single-cell methods that interrogate DNA and RNA simultaneously in the same cell (scWGS-RNA-seq) have been developed ${ }^{23-28}$. However, these first-generation scWGS-RNA-seq methods have not been widely applied since their invention, largely because they require physical separation of DNA and RNA, either by physical separation of the nucleus from the cytoplasm, or by physical separation of mRNA from the rest of the cell by polyT-bead based fishing. Those methods requiring nuclear separation are labor-intensive and technically demanding, time-consuming, requiring well-trained experimental techniques, or require special microfluidic devices ${ }^{29,30}$. They are also not applicable to frozen samples, where it is impossible to obtain intact single-cell suspensions. As such, existing scWGS-RNA-seq methods cannot be applied to the vast majority of primary biobanked tumor samples, which represents most of the readily available clinical samples. G\&T-seq uses polyT beads to capture mRNA from a single cell, followed by separate library preparation of the mRNA and DNA ${ }^{23}$. DR-seq uses reverse transcription (RT) to barcode the RNA in a single cell, then employs quasilinear amplification to pre-amplify both gDNA and RNA together, followed by separation of the reaction into two halves and finishing the amplification and library construction of DNA and mRNA separately from each half ${ }^{24}$. In principle, these two methods can be applied to single nuclei derived from frozen samples, but the need to physically separate DNA and RNA results in lowered data quality, possibly due to loss of materials during the transfer step (Supplementary Fig. 1a-c; Supplementary Note). All in all, these limitations make first-generation scWGS-RNA-seq methods not easily accessible.

We introduce a novel single-cell DNA and RNA co-amplification method, scONE-seq, which enables co-profiling of the transcriptome and genome from the same single cell or nucleus in a one-tube reaction. We devised a barcoding strategy that introduces a 6-base long DNA-specific and RNA-specific barcodes to each type of nucleic acid during the single-cell DNA/RNA amplification process, while also incorporating UMIs ${ }^{31-33}$. Thus, DNA and RNA reads can be distinguished by their barcode information after sequencing. scONE-seq has several advantages: it has a simplified library construction workflow; it is compatible with standard biology workflows such as fluorescence-activated cell sorting (FACS); being a one-pot reaction, its throughput can be easily scaled up using liquid-handling robots while still being easily accessible to manual users; it eliminates transfer steps and the material loss associated with those steps to generate superb data quality. These advantages culminate in a versatile method that generates data of superb quality for both the genome and transcriptome. Importantly, scONE-seq does not require physically separating the DNA and RNA, and is therefore applicable to a variety of sample types including single nuclei. This means that frozen clinical samples and tissue types like liver, bone, and brain, that are difficult to dissociate into single-cell suspensions, which are intractable with many other scWGS-RNA-seq methods, can be profiled using scONE-seq.

We demonstrate that the technical performance of scONE-seq is comparable to existing single-cell methods in a benchmarking experiment using various sample types, including several cell lines and lymphocytes from the blood of a healthy donor. We then show how scONE-seq can be used to discover novel disease-related information by applying it to a frozen IDH1-mutant astrocytoma sample. Using scONE-seq data, we were able to simultaneously characterize both the molecular phenotype and genotype heterogeneity of this patient sample, profiling the cell types in the tumor as well as the clonal events. We were able to fully dissect the genome level and transcriptomic level heterogeneity of this patient's disease, and in doing so we identified a unique normal-like tumor clone by integration of clonal and transcriptomic information. Further analysis of RNA and DNA profiles of this clone suggests that this subpopulation may play a role in the maintenance of the tumor, as well as in regulating the tumor's interactions with normal cells in its microenvironment. The abnormal genomic profile of this small subpopulation along with its normal-like transcriptomic phenotype hints at its origins during the tumor initiation process and demonstrates the power of scONE-seq to distinguish genomic variations of near-normal cells from true normal cells. Using this frozen primary glioma sample 
as an example, we establish a framework for integrative single-cell multi-omic WGS/RNA-seq analysis on biobanked samples to generate new insights on tumor heterogeneity, enabled by the versatility and scalability of the scONE-seq approach.

\section{Results}

\section{Molecular barcoding strategy enables accurate and sensitive co-profiling of DNA and RNA from a single cell in a one-tube reaction}

To achieve single-cell genome and transcriptome co-profiling, we devised a workflow to amplify RNA and DNA simultaneously (Fig. 1a). Briefly, to perform scONE-seq, after the sample dissociation, cells or nuclei are sorted into PCR plates containing lysis buffer with a flow cytometer. Plates of sorted single cells can then be processed immediately, or store at -80 for months before processing. To start the single-cell amplification, we first use Tn 5 with a custom adaptor to fragment and label the genome or any other DNA within the cells ${ }^{34-36}$. In this step, the amplification adaptor, which includes a 6-nucleotide "DNA barcode" and 6-nucleotide UMI, is added to fragmented DNA (fDNA). Subsequently, we use reverse transcription (RT) to generate cDNA, where the RT primer is comprised of: a priming sequence adapted from that of the MATQ-seq protocol37; a 6-nucleotide "RNA barcode"; and a 5-nucleotide UMI. The RT priming sequence is a modified random oligo and primes to the internal regions of RNA transcripts, thereby enabling detection of full-length transcripts, including nonpolyadenylated (non-polyA) RNAs. cDNA 3' adaptor is then added through subsequent poly-C tailing and degenerate PCR ${ }^{38,39}$. Once DNA-specific and RNAspecific barcodes have been added, fDNA and CDNA are amplified simultaneously, and the sequencing library is constructed with the pre-amplified products (Methods).

First, to characterize the transcriptome generated by scONE-seq, we benchmarked it against Smart-seq2 (SS2) $)^{40,41}$ using a variety of test samples: extracted RNA-free E.coli genomes (mock DNA), extracted DNA-free human total RNA (mock RNA), as well as a mixture of the two (i.e. E. coli DNA mixed with human total RNA); and cultured HCT116 single cells. We evaluated the sensitivity by assessing the number of genes detected in each of the benchmark mock and HCT116 samples and found that scONE-seq detected more genes per cell than SS2 (Fig. $1 \mathrm{~b}$ and Supplementary Fig. $1 \mathrm{~d}, \mathrm{P}<2 \times 10^{-16}, \mathrm{t}$-test). This is likely due to scONE-seq being able to capture total RNA 37,42 while SS2 only targets polyA RNA, and therefore capturing a more diverse set of molecules at any given sequencing depth (Supplementary Fig. 1e). Also, scONE-seq enables full-length transcript profiling and achieves gene body coverage uniformity comparable to SS2 (Fig. 1c). We then used sample-to-sample correlation analysis as well as the detection of ERCC Spike-In to estimate the accuracy of scONE-seq in comparison to SS2. The sample-to-sample correlation analysis shows the two methods are comparable (Fig. 1d, Supplementary Fig. 1f). Using ERCC spike-in as a measure of accuracy and sensitivity, SS2 and scONE-seq have comparable performance in terms of both correlation and limit of detection when validating detected quantities against the expected ERCC concentrations; both are sufficiently high for quantitative measurement of transcript abundance from single cells (Supplementary Fig. 1c).

Next, we sought to validate the whole genome sequencing (WGS) capability of scONE-seq. Lorenz curves ${ }^{43}$ compare the coverage uniformity for each method, showing a superior performance by scONE-seq (Fig. 1e; Supplementary Fig. 1a). Then, we used bulk HCT116 WGS data in comparison to scWGS data generated by scONE-seq to confirm that CNAs captured by scONE-seq are consistent with those defined by bulk ( $5 \times 10^{6}$ cells) and pseudo bulk ( 86 cells) (Fig. 1f, g, h). In addition, we performed UMI deduplication on the scONE-seq DNA dataset, since our method adds UMI to the DNA fragments during the tagmentation step, to reduce the bias introduced during single-cell DNA amplification (Methods).

Summarily, the analysis of scRNA-seq and scWGS data generated using benchmark samples shows that scONE-seq can profile genome and transcriptome data from the same single cell without compromising data quality as compared to existing standard methods.

\section{scONE-seq data correctly assigns cell types from primary donor samples}

After thoroughly assessing the technical performance of scONE-seq, we next applied it to known biologically heterogeneous samples to evaluate whether it can accurately identify cellular subtypes within a mixed population. To do so, we performed scONE-seq on four different cell lines, as well as on a primary peripheral blood mononuclear cell (PMBC) sample from a healthy donor.

First, we analyzed the cell-line dataset containing 86 HCT116 cells, 143 NPC43 cells, 37 HUVEC cells, and 17 H9 cells to check for accurate cell-type assignment. With unsupervised graph-based clustering of the RNA expression data, cells from the same cell lines successfully clustered together (Fig. 2a). We also checked the gene markers for each cell line (Fig. 2b) and notably, several well-studied gene markers for these cell lines are found in the scONE-seq dataset (Fig. 2b, Supplementary Fig. 2a).

Next, we used lymphocytes from PBMC to test scONE-seq cell-types clustering accuracy in primary samples. We prepared sequencing libraries with scONE-seq and SS2 from the same PBMC sample for comparison. After quality control filtering to remove low-quality cells, we collected 200 cells for scONE-seq and 194 cells for SS2. With unsupervised graph-based clustering, we found no difference in the cell-type composition between the two methods (Fig. $2 \mathrm{c}, \mathrm{e}, \mathrm{P}=0.5109$, Chi-squared test). After clustering, we annotated the cell types using known lymphocyte markers ${ }^{44-46}$ (Fig. 2 d,f, and Supplementary Fig. 2 b): B cells distinguished by CD19 and MS4A1 (CD20); T cells characterized by CD3E; Less differentiated T cells (Naïve and memory T cells) identified by SELL (CD62L), CCR7, and LEF1; CD4+ T cells characterized by CD4; CD8+ T cells characterized by CD8A and CD8B; and cytotoxic T cells distinguished by PRF1 and NKG7. Notably, cytotoxic T cells comprise both Gamma delta T cells ( $\gamma \delta$ T cell; expressing TRDC, TRGC1, and TRGC2; Supplementary Fig. 2c) as well as effector memory T cells (TEM; lacking CCR7 expression and positively expressing IL2RB) (Supplementary Fig. 2b). We surmised that these are TEM rather than effector T cells since the sample is from a healthy donor. Beyond that, in the scONE-seq dataset, we also captured some regulatory T cells (Treg cell; FOXP3+, CCR4+) (Supplementary Fig. 2d) and detected several non-polyA genes including PZP and SESN3 whose expressions in T-cells have previously been described $^{47}$ (Supplementary Fig. 2e); these features were not found in the SS2 dataset.

These results collectively demonstrate that scONE-seq RNA data can accurately capture the biological variation within a heterogeneous sample. 
The analysis above shows the feasibility of scONE-seq for the cell-type assignment using RNA data. Next, we evaluated the performance of clone identification with scONE-seq WGS data. Here, we utilized scONE-seq WGS data that was obtained simultaneously from the cell lines used in the previous celltype assignments analysis, and delineated the CNAs clonal structure of all four cell lines, followed by hierarchical clustering with their integer copy number profiles (Fig. 2g). From this analysis, we see that HCT116 maintains a relatively homogeneous clonal composition, whereas NPC43, a primary patient-derived cell line that shows strong genome instability, is comprised of 3 main clones (Fig. 2g). Furthermore, the CNAs structure of these 3 clones differ substantially compared to when the cell line was first established ${ }^{48}$ (Fig. 2h, Supplementary Fig. 3a, b, c, d), especially in chromosomes 1, 3, 4, 6, 7, and 11. Correspondingly, distinctions between clones are mainly found in chromosomes 1, 3, 7, and 11 (Supplementary Fig.3a, b, c, d). Based on this observation, the change in chromosome copy numbers during cell culturing of primary cell lines could be a common phenomenon in cell lines with abundant CNAs and unstable genomes. Studies have shown extensive genetic variation across different cell culture lines, and that single cells from some cell lines can give rise to populations with multiple clones due to genome instability ${ }^{21,49}$. Additionally, with the matched transcriptomes of every single cell and their corresponding copy number states, we mapped the clonal information to the transcriptome UMAP for NPC43 and found that the CNAs in NPC43 did not impact the transcriptome state dramatically (Supplementary Fig. 3e). This demonstrates that scONE-seq can identify both phenotype and genotype states for each individual cell.

\section{Dissecting the clonal structure and cell-type subpopulations of an IDH1-mutant astrocytoma}

Gliomas, especially high grade ones, are some of the most aggressive malignant tumors originating in the brain ${ }^{50,51}$. When studying gliomas or other brain tissues using single-cell technology, it is challenging to obtain intact dissociated whole single cells, especially cells with complex morphology, and could lead to biases in cell-type sampling ${ }^{52}$. As such, for brain single-cell profiling, single nucleus isolation is more widely used. To profile both the genotypic and phenotypic heterogeneity in a biobanked sample, we apply scONE-seq on single nuclei isolated from a snap-frozen astrocytoma specimen that has been stored since the surgery for two years prior to analysis: a second recurrent (2R) astrocytoma sample with IDH1 (R132H), TP53 (P278S), and ATRX (R781*) mutations (Supplementary Fig. 4a). The primary (P) and first recurrent (1R) samples were limited in quantity, and subject to whole exome sequencing (WES) and RNA sequencing in bulk ${ }^{53}$ (Fig. 3a). In total, we used scONE-seq to profile over 1,200 nuclei, including 1,210 scRNA datasets, 1,089 scWGS datasets, generating 908 passed-QC paired DNA and RNA datasets.

First, we delineated the clonal structure of this 2R astrocytoma sample. Using dimension reduction on scWGS data, we clustered cells into four distinct genomic states (Fig. 3b), consisting of one cluster of normal cells and three tumor clones (Supplementary Fig. 5a). Whole genome duplication was also found in this tumor and validated by measuring each cell's DAPI intensity using flow cytometry (Supplementary Fig. 5b). We were also able to identify aneuploidy and loss of heterozygosity in multiple loci using the B-allele frequency estimation (Supplementary Fig. 5c, d), and the integer copy number of cells was subsequently calculated (Fig. 3c). Based on the genomic profile of each clone and the WES data from the primary and recurrent tumors, the phylogenetic tree of the patient was constructed. The $2 \mathrm{R}$ clone 1 was found to be closer to the root (normal cell), with fewer loss of heterozygosity (LOH) events, and it has very similar genome alterations as the primary tumor WES data (Fig. 3d, e; Supplementary Fig. 5e). The 2R clone 2 and clone 3 harbor many of the same deletion regions as the $1 \mathrm{R}$ tumor, resulting in $\mathrm{LOH}$ (Fig. 3e; Supplementary Fig. $5 \mathrm{e}$ ). We surveyed the known driver genes and found $B R A F, M E T$, and $M Y C$ to be amplified in all the $2 \mathrm{R}$ clones ${ }^{53-55}$ (Fig. 3b). Several key deletion events were found to only occur in the $2 \mathrm{R}$ clone 2 and clone 3 , including deletion of $C D K N 2 A$, and PTEN; in particular, the homozygous deletion of $C D K N 2 A$ is a known prognostic factor for IDH mutant astrocytomas, and was recently incorporated into the World Health Organization central nervous system tumors classification scheme as a sufficient criterion for classifying cases as Grade 4, even when classical histology criteria are not fulfilled ${ }^{56}$ (Supplementary Fig. 5f).

Next, we analyzed the RNA data from this dataset. First, we performed unsupervised graph-based clustering on scONE-seq RNA data, obtaining multiple cell clusters that were then annotated based on their RNA markers. We found this tumor contains tumor-associated macrophages (TAM), neurons, astrocytes, oligodendrocytes, and tumor cells defined by their canonical cell type gene signatures (Fig. 4a, b). The complex tumor microenvironment (TME) indicates the highly infiltrated phenotype of this recurrence tumor. The tumor cells - those that have transcriptomes classified as tumor-like - display high EGFR expression, a well-known feature of high grade gliomas. These tumor cells can further be subset into 4 cellular states based on meta-module scores described by Neftel et al. ${ }^{13}$ (Fig. 4a): oligodendrocyte progenitor cell-like (OPC-like), neural progenitor cell-like (NPC-like), mesenchymal-like (MES-like), and astrocyte-like (AC-like) cellular states.

In addition to the phylogenetic tree obtained from DNA data, which dissects the clonality, we are also able to use paired RNA data to superimpose the cell-type information onto the clonal information to identify clonal subpopulations with unique functional, phenotypic features. To do so, we mapped the clonal information to the RNA UMAP to visualize the clonal distribution among different cell types (Fig. 4c). The $2 \mathrm{R}$ clone 3 is the major clone of this tumor and is differentiated into all 4 tumor phenotypes: OPC-like, NPC-like, MES-like, and AC-like cellular states. The 2 R clone 2 consists predominantly of AC-like cells. The 2R clone 1 is the most interesting: using RNA data alone, all cells from this clone were clustered with normal astrocytes, indicating transcriptome similarity between $2 \mathrm{R}$ clone 1 and normal astrocytes that is indistinguishable using scRNA-seq data alone; but upon superimposing matched genotype and phenotype information, this unique population of astrocyte-like tumor cells with clearly abnormal genotype as compared to the true normal cells are revealed (Fig. 4c). This highlights the importance of directly measuring copy number profiles as a standard to identify cancer cells and is particularly important in the study of tumor initiation for capturing the normal-to-cancer transition.

Next, we sought to validate the presence of this clone within the tumor and to investigate its potential role in the tumor microenvironment.

Characterization of a unique tumor clone with normal astrocyte-like phenotype 
To verify the existence of clone 1 cells, we first identified gene markers unique to clone 1 , including $X I S T, R F X 3, A D C Y 8$, and GRIA1, which can distinguish them from other tumor and normal cells (Fig. 4d, Supplementary Fig. 6a). These markers were also found in the droplet-based snRNA-seq dataset to label a putative clone 1 population, also adjacent to normal astrocytes (Supplementary Fig. 6b, c, d). As validation, we integrated the scONE-seq RNA dataset and 10X dataset to show that our scONE-seq dataset of 1210 nuclei exhaustively captured all cell types that were observed in droplet snRNA-seq of 4416 nuclei and showed that clone 1 merged with the putative clone 1 cells from the droplet-based snRNA-seq dataset (Fig. 4e).

Then, we performed histological analyses on FFPE sections from both the primary and 2R tumors of this patient to identify the cells with IDH1 (R132H) and ADCY8. Anti-IDH1 (R132H) is expected to label all tumor cells, as this is known to be a somatic mutation in IDH-mutant glioma cells ${ }^{55,57,58}$ (Supplementary Fig. 4a), and anti-ADCY8 is expected to mark some normal neurons and normal astrocytes in addition to clone 1 cells (Fig. 4d, Supplementary Fig. 6e). As such, putative clone 1 cells are those cells marked by double-positive staining of IDH1 (R132H) and ADCY8. First, we looked at the overall staining pattern across the whole slide section and noted that the IDH1 (R132H) positive tumor cells are distributed over the entire section for both the primary and $2 \mathrm{R}$ tumors. The ADCY8 signals appear stronger in $2 \mathrm{R}$ tumor sections and are specifically concentrated to certain regions which also express IDH (R132H) more strongly (Supplementary Fig. 7a). Interestingly, these ADCY8 positive regions are always near the IDH1 (R132H) negative 'normal adjacent' regions (Supplementary Fig. 7a). The double-positive cells that we suspect to be the putative clone 1 cells appear to be near other normal and malignant cells (Fig. 5a). These histological immune staining results provided additional details on the spatial distribution of putative clone 1 cells in the tumor sections.

The presence of these clone 1 tumor cells with their normal-like transcriptional signature among normal astrocytes and neurons prompted us to examine the gene expression of clone 1 cells associated with signaling and cell-cell communication. Several studies have demonstrated that glioma cells can form synaptic structures with normal neurons as a signaling conduit within the tumor ${ }^{59-63}$. Specifically, this was found to occur via tumor microtubes displaying AMPA receptors (AMPAR), a glutamate receptor subtype ${ }^{62,63}$. AMPARs are tetrameric, and there are four subunit proteins involved, Glut1-4, encoded by the genes GRIA1-4 respectively ${ }^{64,65}$. Interestingly, we found the GRIA1 genes to be differentially expressed between the different tumor clones in our sample (Fig. $5 b)$. The major clone, clone 3, expressed GRIA2-4 and does not express GRIA1; clone 1, however, is the only tumor subpopulation that expresses GRIA1 and all three other GRIA family genes are expressed at much lower levels. The gene expression feature of clone 1 generally resembles astrocytes, including the expression of the astrocytic marker APOE (Supplementary Fig. 7b), but normal astrocytes do not express GRIA1 (Fig. 5b, Supplementary Fig. 7b). Next, we also performed ligand-receptor analysis for the different subpopulations and found TGF $\beta$ signaling transcripts to be strongly and specifically expressed in normal astrocytes, clone 1 cells, and TAMs (Supplementary Fig. 7c), with clone 1 cells expressing the ligand and predominantly the TAMs expressing the receptor (Fig. 5c). These observations hint at the potential role of clone 1 cells within the tumor, and future studies expanded to additional patient samples will be necessary to determine the generalizability of these phenomena.

\section{Discussion}

We have developed the versatile scONE-seq method, which is comparable with previous methods in multiple aspects including sensitivity and accuracy, and surpasses previous methods on throughput, versatility, and sample applicability (Supplementary Note). Most importantly, we designed this technology to be compatible with frozen tissue samples that have been stored for years. This feature makes it easier to plan and perform larger-scale clinical multi-omic singlecell studies in two ways: First, by enabling studies on existing biobanked samples, which we have demonstrated herein; Second, for studies on new samples, it also removes the burden of having to immediately process tissues from clinical researchers whose priority is patient care. The application of scONE-seq on frozen tumor tissue in our study demonstrates a comprehensive investigation of the genotypic and molecular phenotypic heterogeneity of astrocytoma. By superimposing the multi-omic data, we directly observed and characterized the differentiated tumor clones, which supports the idea that tumor clones can produce a differentiation hierarchy $7,66,67$. In addition, we found a unique tumor clone in this patient's $2 \mathrm{R}$ astrocytoma with a very similar transcriptomic phenotype as normal astrocytes. Putative clone 1 cells expressing the same gene markers as clone 1 were found using both independent $10 \mathrm{X}$ Genomics snRNA-seq as well as immunostaining on tissue sections. Incorporating the histological and transcriptomic information of clone 1 cells, we propose some potential roles of these cells in the TME, including cell-cell communication via both signaling molecules and potential tumor-neuron synapse formation. Specifically, we found clone 1 to be the only tumor subpopulation that expresses GRIA1, an AMPA receptor encoding gene, while all the other tumor clones only express other GRIA genes. GRIA1-encoded GluA1 subunit often forms GluA1 homomer AMPARs, which are calcium-permeable and broadly found in synapses in early development ${ }^{65}$. Calcium-permeable AMPARs are a key signaling molecule in the tumor-neuron synapse, and the maintenance of long-term potentiation is also known to be modulated through post-translational modification of GluA1, making GluA1 critical for neural plasticity in the brain ${ }^{65,68}$. The unique, differential expression of GRIA1 in clone 1 hints at a unique, possibly multi-faceted role for clone 1 in cell-cell communication in the tumor microenvironment ${ }^{7,62,63}$. We also found TGF $\beta$ signaling expression to be prominently expressed in normal astrocytes, clone 1 cells, and TAMs. TGF $\beta$ signaling in glioblastomas can promote invasiveness, angiogenesis, and plays a role in the suppression of the immune system; it is a therapeutic target for the treatment of gliomas. The expression of TGF $\beta$ pathway-associated genes by clone 1 cells is comparable to that of the normal astrocytes within the tumor, raising intriguing questions about the role of clone 1 in tumor immune modulation, and its possible interactions with TAMs. These features of the tumor microenvironment and whether clone 1 cells are a commonly occurring cell-type in gliomas or specifically astrocytomas will be an interesting avenue of future investigation on a larger sample set. Collectively from these results, we posit that scRNA-seq-only based cancer studies could underestimate important layers of tumor heterogeneity, and that simultaneous direct DNA measurement could contribute meaningful and informative insight on tumor evolution. Meanwhile, the clonal analysis based on scWGS-only data also ignores the complex interactions within a tumor microenvironment. By deciphering the genetic and phenotypic heterogeneity within the tumor ecosystem with scONE-seq, we can reveal the interplays of clonal expansion, tumor cells differentiation hierarchy, and TME. We anticipate that future large-scale studies on more samples will be even more informative, and has the potential to inform our knowledge of cancer and therapeutics in a more generalizable manner.

Initially, scONE-seq suffered cross-contamination from DNA to RNA due to mislabeling of barcodes; this problem was resolved by optimizing the reverse transcription temperature and amplification extension time (Supplementary Table 1). Further, we checked the mapping region of the RNA data from single 
cells with DNA and RNA co-profiles and single cells with only RNA profiles. The exon mapping proportion difference is inconsequential (Supplementary Fig. 1e), suggesting the mislabeled molecules are a negligible fraction of the total sequenced reads. Further benchmarking and validation experiments with different sample types demonstrated that scONE-seq data correctly identifies cell types and clones simultaneously.

Benefitting from its one-tube reaction system, scONE-seq possesses very high scalability, and a higher throughput version can be achieved by simply adapting this method to a robotic liquid handler. Alternatively, producing scONE-seq and droplet-based single-cell data in parallel and then integrating them, is also a useful complementary, multi-omics approach to study cancer with high throughput. Moreover, additional processing can be easily added to the scONE-seq workflow to enable profiling of more layers of information: To detect chromatin accessibility simultaneously, an additional nuclei tagmentation step ${ }^{69-71}$ with customized ATAC adaptors could be added before FACS sorting; Similarly, quantitative protein estimation ${ }^{72}$ could be achieved by using DNA-barcoded antibodies before single-cell sorting steps of the scONE-seq; and by jointly performing whole-exome capturing or any hybridized target sequencing panels with the standard scONE-seq library, paired high-depth single-cell somatic mutation information could also be integrated into the scONE-seq dataset. In general, scONE-seq has very strong scalability to measure different signals from a cell. We expect that scONE-seq will be a powerful tool to dissect cellular heterogeneity and will inspire other ultra-high-throughput single-cell multi-omics methods development.

\section{Methods}

Single cell or nucleus isolation. HCT116 (ATCC), NPC43 (kindly provided by Prof. KW Lo in Chinese University of Hong Kong), HUVEC (LONZA), and H9 (WiCell) cells are dissociated with trypsin-EDTA ( $0.25 \%$ ) solution (Thermo Fisher) and stained with propidium iodide ( $1 \mathrm{mg} / \mathrm{ml}$, Thermo Fisher) to exclude dead cells.

Fresh whole blood was taken in the HKUST clinic from a healthy human donor. Lymphocytes were isolated via Ficoll-Paque PLUS (GE Healthcare) density centrifugation. The red blood cells were removed with 1X Red Blood Cell lysis buffer (Thermo Fisher).

The frozen IDH1-mutant astrocytoma tissue (stored at $-80^{\circ} \mathrm{C}$ ) was obtained from Prince of Wales Hospital. The nuclei isolation protocol is based on previous studies $^{73,74}$. In brief, the homogenization method was used to prepare nuclei. $100 \mathrm{mg}$ frozen tissue was put into pre-chilled glass douncer contained $2 \mathrm{ml}$ of $1 \mathrm{X}$ homogenization buffer $\left(5 \mathrm{mM} \mathrm{CaCl}_{2}, 3 \mathrm{mM} \mathrm{Mg}(\mathrm{Ac})_{2}, 10 \mathrm{mM}\right.$ Tris, $167 \mu \mathrm{M} \beta$-mercaptoethanol, $320 \mathrm{mM}$ sucrose, $0.1 \mathrm{mM}$ EDTA, 0.1\% NP40, $1 \mathrm{U} / \mathrm{ml}$ RNase inhibitor, $\mathrm{pH}=7.8$ ). The 'loose' and 'tight' pestles were applied to homogenize the frozen tissue. The homogenized suspension was then filtered with a $40 \mu \mathrm{m}$ cell strainer (Corning), and nuclei can be spun down at $1000 \mathrm{~g}, 10 \mathrm{mins}, 4^{\circ} \mathrm{C}$. We utilized Sucrose Density Gradient to remove cell debris. Nuclei were resuspended in $3.0 \mathrm{ml}$ low sucrose buffer ( $320 \mathrm{mM}$ sucrose, $10 \mathrm{mM} \mathrm{HEPES}, 5 \mathrm{mM} \mathrm{CaCl}, 3 \mathrm{mM} \mathrm{Mg}(\mathrm{Ac})_{2}, 0.1 \mathrm{mM}$ EDTA, $1 \mathrm{mM} \mathrm{DTT}, 0.2 \mathrm{U} / \mathrm{ml}$ RNase inhibitor, $\mathrm{pH}=8.0$ ). We then layered $12.5 \mathrm{ml}$ of density sucrose buffer ( $1 \mathrm{M}$ sucrose, $10 \mathrm{mM} \mathrm{HEPES}, 3 \mathrm{mM} \mathrm{Mg}(\mathrm{Ac})_{2}, 1 \mathrm{mM} \mathrm{DTT}$, pH=8.0) underneath the low sucrose buffer homogenate to centrifuge at $3200 \mathrm{~g}$ for $20 \mathrm{~min}$ at $4^{\circ} \mathrm{C}$. After removing the supernatant, nuclei were resuspended in sorting buffer (1XPBS, $1 \% \mathrm{BSA}, 1 \mathrm{U} / \mathrm{ml}$ RNase inhibitor, $0.1 \mathrm{mg} / \mathrm{ml}$ DAPI (Thermo Fisher)).

Cells or nuclei were then loaded to Aria III flow cytometer (BD Biosciences) to sort single cells into PCR tubes (96 or 384 PCR plates) containing lysis buffer. The lysis buffer consisted of $2.5 \mathrm{U} / \mu \mathrm{l}$ RNase Inhibitor (NEB), $0.15 \%$ Triton X-100 (Sigma), and $6 \mu \mathrm{M}$ DTT (Thermo Fisher). The sorted sample can be stored at $-80^{\circ} \mathrm{C}$ for months.

Generation of scONE-seq libraries. To start the scONE-seq pre-amplification, the proteinase K (Sigma) was used to completely lyse cells or nuclei. Tagmentation reaction was performed to fragment the genome DNA and add the DNA-specific barcode. This reaction includes the following components, 6 $\mathrm{mM} \mathrm{MgCl} 2,0.5 \mathrm{mM}$ dNTP (NEB), $8.5 \mathrm{mM}$ TAPs- $\mathrm{NaOH}, 1.5 \mathrm{U} / \mu \mathrm{l}$ RNase Inhibitor, $0.05 \mathrm{U}$ KAPA polymerase (Roche), $8 \%$ PEG8000, and One-Tn5 ${ }^{35,36}$ with customed adaptor (GTCTCGTGGGCTCGGTCATGN ${ }_{5}$ AGATGTGTATAAGAGA CAG) (Novoprotein). The reaction was incubated at $55^{\circ} \mathrm{C}$ for 10 mins followed by $72^{\circ} \mathrm{C}$ for 10 mins. Then, proteinase $\mathrm{K}$ or thermolabile proteinase $\mathrm{K}$ (NEB) was used to deactivate the enzyme in the buffer. Thereafter, we performed reverse transcription with the following components, $40 \mathrm{U}$ SuperScript ${ }^{\mathrm{TM}}$ III Reverse Transcriptase (Thermo Fisher), $70 \mathrm{mM} \mathrm{Tris-HCl,} 1.5 \mathrm{U} / \mu \mathrm{RNase}$ Inhibitor, $8 \mathrm{mM}$ $\mathrm{MgCl}_{2}, 7 \mu \mathrm{M}$ DTT and $0.15 \mu \mathrm{M}$ RT primers (GTCTCGTGGGCTCGGATCGTN ${ }_{5}{ }_{20} \mathrm{VN}$; GTCTCGTGGGCTCGGATCGTN ${ }_{5}$ GGGHN;

GTCTCGTGGGCTCGGATCGTN ${ }_{5}$ TTTVN). Reverse transcription was carried out at $12^{\circ} \mathrm{C}$ for 12 sec followed by gradient increasing to $50^{\circ} \mathrm{C}$, and incubating for $50 \mathrm{~min}$, and $55^{\circ} \mathrm{C}$ for $50 \mathrm{~min}$. Subsequently, the residual primers and RNA were removed with thermolabile EXO I (NEB), RNase If (NEB), and RNase $\mathrm{H}$ (NEB). Then, the terminal transferase (NEB) was used to add the C-tail to cDNA fragments. This reaction was performed at $37^{\circ} \mathrm{C}$ for 5 mins and the enzyme was immediately deactivated with thermolabile proteinase K. Second strand synthesis was then performed by adding, $0.3 \mu \mathrm{M} 3^{\prime}$ adaptor (GTCTCGTGGGCTCGGAT $\left.\mathrm{CGTN}_{5} \mathrm{GGGHN}\right), 1 \mu \mathrm{K}$ KAPA HIFI Fidelity Buffer (5X), $0.7 \mathrm{mM}\left(\mathrm{NH}_{4}\right)_{2} \mathrm{SO}_{4}$, and $0.1 \mu \mathrm{KAPA}$ Polymerase. The reaction was incubated at $72^{\circ} \mathrm{C}$ for $5 \mathrm{~min} ; 10 \mathrm{cycles}$ of $\left(1 \mathrm{~min}\right.$ at $48^{\circ} \mathrm{C} ; 1 \mathrm{~min}$ at $\left.72^{\circ} \mathrm{C}\right)$; and $5 \mathrm{~min}$ at $72^{\circ} \mathrm{C}$, in a thermal cycler. Additional residual primers removal reaction was performed with Exo I (NEB). Lastly, $14 \mu \mathrm{l} \mathrm{KAPA} \mathrm{HotStart} \mathrm{ReadyMix} \mathrm{(2X),} 1.5 \mathrm{mM}\left(\mathrm{NH}_{4}\right)_{2} \mathrm{SO}_{4}, 2 \%$ DMSO (Thermo Fisher), $1.2 \mu \mathrm{M}$ amplification primer (GATGTGTGGAGGTCTCGTGGGCTCGG) was added to amplify DNA and RNA simultaneously. The PCR was performed at $98^{\circ} \mathrm{C}$ for $4 \mathrm{~min} ; 18-20$ cycles of $\left(20 \mathrm{~s}\right.$ at $98^{\circ} \mathrm{C} ; 4.25 \mathrm{~min}$ at $\left.72^{\circ} \mathrm{C}\right)$; and $10 \mathrm{~min}$ at $72^{\circ} \mathrm{C}$, in a thermal cycler. All primer sequences can be found in Supplementary Table 2.

scONE-seq sequencing libraries construction. Pre-amplified samples were purified with Ampure XP beads (Beckman). Samples were diluted to $0.1 \mathrm{ng} / \mu \mathrm{l}$ and performed tagmentation reaction with the following components, 1 X TAPs buffer ( $50 \mathrm{mM}$ TAPS- $\mathrm{NaOH}, 25 \mathrm{mM} \mathrm{MgCl}, \mathrm{pH}=8.0$ ), $8 \%$ PEG8000, $0.001 \mu \mathrm{P} 5$ Tn5 (Adaptor sequence: TCGTCGGCAGCGTCAGATGTGT ATAAGAGACAG). The reaction was performed at $55^{\circ} \mathrm{C}$ for $15 \mathrm{~min}$. Samples were then amplified with Illumina sequencing index primers (Supplementary Table 2) (Sangon) by using KAPA HiFi HotStart Polymerase Kit (Roche). The enrichment PCR was incubated at $95^{\circ} \mathrm{C}$ for $10 \mathrm{~min} ; 10-11$ cycles of $\left(20 \mathrm{~s}\right.$ at $98^{\circ} \mathrm{C} ; 15 \mathrm{~s}$ at $60^{\circ} \mathrm{C} ; 30 \mathrm{~s}$ at $\left.72^{\circ} \mathrm{C}\right)$; and 2 min at $72^{\circ} \mathrm{C}$, in a thermal cycler. Samples were then pooled and purified with Ampure XP beads. scDASH protocol was then used to remove the abundant ribosome and mitochondrial sequences ${ }^{75,76}$. Double size selection can be performed to optimize the library size. The library was then sequenced on Illumina NextSeq500 with customized sequencing primers (Supplementary Table 2). 
DNA and RNA data separation. Sequencing data were firstly filtered with fastp ${ }^{77}$. Fastq files were then separated into DNA fastq files, RNA fastq files, and Unmatched fastq files with seqkit, seqtk, and bbduk ${ }^{78-80}$. During this process, UMI of the reads was extracted and labeled to fastq files head with fastp ${ }^{77}$.

DNA data analysis. DNA fastq files were mapped to hg38 (ftp://ftp.ncbi.nlm.nih.gov/ genomes/all/GCA/000/001/405/GCA_000001405.15_GRCh38/seqs_for_alignment_pipelines.ucsc_ids/GCA_000001405.15_GRCh38_no_alt_analysis_set.fna.! with BWA mem ${ }^{81}$. To perform UMI-based deduplication, read2 reads in bam files were extracted with samtools ${ }^{82}$ and deduplicated with umi_tools ${ }^{83}$. The deduplicated read2 reads were used to extract its paired read1 and these paired fastq were then re-aligned to hg38 with BWA mem ${ }^{81,84}$.

If only performing the counts-based copy number variation analysis, Ginkgo was used to generate the normalized counts ${ }^{43}$. If performing the allele-specific copy number variation analysis, CHISEL was used to generate allele frequency information ${ }^{85,86}$. The integer copy number calculation was based on the previous studies $21,22,87$. In this pipeline, the segmentation was performed with copynumber and aCGH in $\mathrm{R}^{88}$.

WES data from different tumor samples were analyzed with CNVkit and THetA to infer the copy number profiles ${ }^{89-91}$. The integer copy number data from WES and scONE-seq were combined to perform the phylogenetic reconstruction, adopted from a previous method $21,92,93$.

RNA data analysis. UMI-based deduplication was also performed with RNA fastq files. The workflow kept the same except replacing the BWA with STAR ${ }^{94}$. Then, the fastq files can be quantified with Kallisto ${ }^{95}$ (cDNA quantification) or Salmon ${ }^{96}$ (pre-mature RNA quantification). 10x snRNA-seq data was quantified with kb-python ${ }^{97}$. The expression data were analyzed using Seurat with sctransform pipeline (normalization, dimension reduction, dataset integration, finding clusters, differential gene analysis) ${ }^{98-100}$. The astrocytoma cellular states scoring was performed following the original paper ${ }^{13}$. The ligand-receptor analysis was performed with CellChat ${ }^{101}$.

Visualization. Plots were created using the ggplot2 and ggtrees R package $\mathrm{e}^{102-104}$. Heatmaps were created with the ComplexHeatmap package ${ }^{105}$. Figures were prepared in Inkscape ${ }^{106}$.

IHC analysis. Slides were obtained from Prince of Wales Hospital. Xylene and ethanol were used to remove wax. Antigen retrieval was performed with Sodium Citrate Buffer (Thermo Fisher) at $98^{\circ} \mathrm{C}$ for 15 min. Blocking was performed in $10 \%$ normal serum (goat and donkey, Abcam) with $1 \%$ BSA in PBST buffer (0.05\% Triton X-100). IDH1(R132H) antibody (Dianova, 1:40) and ADCY8 antibody (Abcam, 1:200) were added to slides and incubated at $4^{\circ} \mathrm{C}$ overnight in a humid box. Secondary antibodies (1:400,anti-mouse, anti-rabbit, Thermo Fisher) were used to provide the fluorescent signal. Mounting buffer with DAPI (Abcam) was used to stain the nucleus and retain fluorescence. Images were taken with Zeiss Axio Scan.Z1 Slide Scanner with $20 X$ objective (Zeiss).

\section{Declarations}

\section{ACCESSION CODES}

The data generated in this study have been deposited into the NCBI Gene Expression Omnibus with accession code GSE185269.

\section{ACKNOWLEDGEMENTS}

The authors thank Prof. Yanyi Huang (Peking University) and Dr. Jianbin Wang (Tsinghua University) for their on-going support and advice on this project. We also thank Prof. Tom H. Cheung (HKUST) for providing us with Tn5 enzyme that was used in a portion of the experiments, and Prof. Kwok Wai Lo (CUHK) for providing the NPC43 cell line used in the study. We thank Dr. Danny C.Y. Leung (HKUST) for his reading and feedback on the manuscript. We also want to thank Prof. Zilong Wen (HKUST), Prof. Yingying Wei (CUHK), and Prof. Can Yang (HKUST) for their discussion and advice on the data analysis.

\section{AUTHOR CONTRIBUTIONS}

LY and ARW designed the study. LY developed scONE-seq chemistry, generated libraries, performed computational analysis, performed the IHC staining, prepared figures, and wrote the manuscript text. XLW provided input to scONE-seq chemistry, generated libraries, and performed the IHC staining. QHM performed computational analysis. SSTT prepared the nuclei and 10x library. DSCL provided input to the scONE-seq chemistry. AKC, WSP, HKN, and DTC prepare patient samples and prepare the FFPE sections. JGW and ARW supervised the work. LY and ARW wrote the manuscript. All authors edited and approved the final manuscript.

\section{COMPETING INTERESTS}

ARW and LY have filed a USPTO provisional patent on scONE-seq (application number 63/093,368)

\section{References}

1. Wu, A. R., Wang, J., Streets, A. M. \& Huang, Y. Single-cell transcriptional analysis. Annual Review of Analytical Chemistry 10, 439-462 (2017).

2. Gawad, C., Koh, W. \& Quake, S. R. Single-cell genome sequencing: Current state of the science. Nature Reviews Genetics 17, 175-188 (2016).

3. Nam, A. S., Chaligne, R. \& Landau, D. A. Integrating genetic and non-genetic determinants of cancer evolution by single-cell multi-omics. Nature Reviews Genetics 22, 3-18 (2021).

4. Birnbaum, K. D. Power in numbers: Single-cell RNA-seq strategies to dissect complex tissues. Annual Review of Genetics 52, 203-221 (2018). 
5. Villani, A. C. et al. Single-cell RNA-seq reveals new types of human blood dendritic cells, monocytes, and progenitors. Science 356, (2017).

6. Treutlein, B. et al. Reconstructing lineage hierarchies of the distal lung epithelium using single-cell RNA-seq. Nature 509, 371-375 (2014).

7. Venteicher, A. S. et al. Decoupling genetics, lineages, and microenvironment in IDH-mutant gliomas by single-cell RNA-seq. Science 355, (2017).

8. Shami, A. N. et al. Single-Cell RNA Sequencing of Human, Macaque, and Mouse Testes Uncovers Conserved and Divergent Features of Mammalian Spermatogenesis. Developmental Cell 54, (2020).

9. McGranahan, N. \& Swanton, C. Clonal Heterogeneity and Tumor Evolution: Past, Present, and the Future. Cel/ vol. 168 613-628 (2017).

10. Prager, B. C., Xie, Q., Bao, S. \& Rich, J. N. Cancer Stem Cells: The Architects of the Tumor Ecosystem. Cell Stem Cel/ vol. 24 41-53 (2019).

11. Kreso, A. \& Dick, J. E. Evolution of the cancer stem cell model. Cell Stem Cell vol. 14 275-291 (2014).

12. Shaffer, S. M. et al. Rare cell variability and drug-induced reprogramming as a mode of cancer drug resistance. Nature 546, 431-435 (2017).

13. Neftel, C. et al. An Integrative Model of Cellular States, Plasticity, and Genetics for Glioblastoma. Cell 178, 835-849.e21 (2019).

14. Wang, L. et al. The phenotypes of proliferating glioblastoma cells reside on a single axis of variation. Cancer Discovery 9, 1708-1719 (2019).

15. Weng, Q. et al. Single-Cell Transcriptomics Uncovers Glial Progenitor Diversity and Cell Fate Determinants during Development and Gliomagenesis. Cell Stem Cell 24, 707-723.e8 (2019).

16. Müller, S. et al. Single-cell profiling of human gliomas reveals macrophage ontogeny as a basis for regional differences in macrophage activation in the tumor microenvironment. Genome Biology 18, (2017).

17. Pombo Antunes, A. R. et al. Single-cell profiling of myeloid cells in glioblastoma across species and disease stage reveals macrophage competition and specialization. Nature Neuroscience 24, 595-610 (2021).

18. Hara, T. et al. Interactions between cancer cells and immune cells drive transitions to mesenchymal-like states in glioblastoma. Cancer Cel/ 39, 779792.e11 (2021).

19. Zhang, L. et al. Single-Cell Analyses Inform Mechanisms of Myeloid-Targeted Therapies in Colon Cancer. Cell 181, 442-459.e29 (2020).

20. Cheng, S. et al. A pan-cancer single-cell transcriptional atlas of tumor infiltrating myeloid cells. Cell 184, 792-809.e23 (2021).

21. Minussi, D. C. et al. Breast tumours maintain a reservoir of subclonal diversity during expansion. Nature 592, 302-308 (2021).

22. Gao, R. et al. Punctuated copy number evolution and clonal stasis in triple-negative breast cancer. Nature Genetics 48, 1119-1130 (2016).

23. Macaulay, I. C. et al. G\&T-seq: Parallel sequencing of single-cell genomes and transcriptomes. Nature Methods 12, 519-522 (2015).

24. Dey, S. S., Kester, L., Spanjaard, B., Bienko, M. \& van Oudenaarden, A. Integrated genome and transcriptome sequencing of the same cell. Nature Biotechnology 33, 285-289 (2015).

25. Hou, Y. et al. Single-cell triple omics sequencing reveals genetic, epigenetic, and transcriptomic heterogeneity in hepatocellular carcinomas. Cell Research 26, 304-319 (2016).

26. Bian, S. et al. Single-cell multiomics sequencing and analyses of human colorectal cancer. https://www.science.org.

27. Zachariadis, V., Cheng, H., Andrews, N. \& Enge, M. A Highly Scalable Method for Joint Whole-Genome Sequencing and Gene-Expression Profiling of Single Cells. Molecular Cell 80, 541-553.e5 (2020).

28. Guo, F. et al. Single-cell multi-omics sequencing of mouse early embryos and embryonic stem cells. Cell Research 2017 27:8 27, $967-988$ (2017).

29. Oguchi, Y., Ozaki, Y., Abdelmoez, M. N. \& Shintaku, H. NanoSINC-seq dissects the isoform diversity in subcellular compartments of single cells. Science Advances 7, (2021).

30. Abdelmoez, M. N. et al. SINC-seq: Correlation of transient gene expressions between nucleus and cytoplasm reflects single-cell physiology. Genome Biology 19, (2018).

31. Kivioja, T. et al. Counting absolute numbers of molecules using unique molecular identifiers. Nature Methods 9, 72-74 (2012).

32. Klein, A. M. et al. Droplet barcoding for single-cell transcriptomics applied to embryonic stem cells. Cell 161, 1187-1201 (2015).

33. Macosko, E. Z. et al. Highly parallel genome-wide expression profiling of individual cells using nanoliter droplets. Cell 161, 1202-1214 (2015).

34. Reznikoff, W. S. Transposon Tn5. Annual Review of Genetics vol. 42 269-286 (2008). 
35. Picelli, S. et al. Tn5 transposase and tagmentation procedures for massively scaled sequencing projects. Genome Research 24, 2033-2040 (2014).

36. Hennig, B. P. et al. Large-scale low-cost NGS library preparation using a robust Tn5 purification and tagmentation protocol. G3: Genes, Genomes, Genetics 8, 79-89 (2018).

37. Sheng, K., Cao, W., Niu, Y., Deng, Q. \& Zong, C. Effective detection of variation in single-cell transcriptomes using MATQ-seq. Nature Methods 14, 267270 (2017).

38. Tang, F. et al. mRNA-Seq whole-transcriptome analysis of a single cell. Nature Methods 6, 377-382 (2009).

39. Sheng, K., Cao, W., Niu, Y., Deng, Q. \& Zong, C. Effective detection of variation in single-cell transcriptomes using MATQ-seq. Nature Methods 14, 267270 (2017).

40. Picelli, S. et al. Smart-seq2 for sensitive full-length transcriptome profiling in single cells. Nature methods 10, 1096-8 (2013).

41. Picelli, S. et al. Full-length RNA-seq from single cells using Smart-seq2. Nature protocols 9, 171-81 (2014).

42. Fan, X. et al. Single-cell RNA-seq transcriptome analysis of linear and circular RNAs in mouse preimplantation embryos. Genome Biology 16, (2015).

43. Garvin, T. et al. Interactive analysis and assessment of single-cell copy-number variations. Nature Methods vol. 12 1058-1060 (2015).

44. Sun, J. C. \& Lanier, L. L. NK cell development, homeostasis and function: Parallels with CD8 + T cells. Nature Reviews Immunology vol. 11 645-657 (2011).

45. Farber, D. L., Yudanin, N. A. \& Restifo, N. P. Human memory T cells: Generation, compartmentalization and homeostasis. Nature Reviews Immunology vol. 14 24-35 (2014).

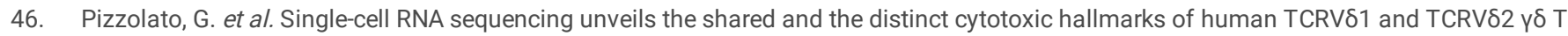
lymphocytes. Proceedings of the National Academy of Sciences of the United States of America 116, 11906-11915 (2019).

47. Uhlen, M. et al. A genome-wide transcriptomic analysis of protein-coding genes in human blood cells. Science 366, (2019).

48. Lin, W. et al. Establishment and characterization of new tumor xenografts and cancer cell lines from EBV-positive nasopharyngeal carcinoma. Nature Communications 9 , (2018).

49. Ben-David, U. et al. Genetic and transcriptional evolution alters cancer cell line drug response. Nature 560, 325-330 (2018).

50. Ceccarelli, M. et al. Molecular Profiling Reveals Biologically Discrete Subsets and Pathways of Progression in Diffuse Glioma. Cell 164, 550-563 (2016).

51. Hu, H. et al. Mutational Landscape of Secondary Glioblastoma Guides MET-Targeted Trial in Brain Tumor. Cel/ 175, 1665-1678.e18 (2018).

52. Habib, N. et al. Massively parallel single-nucleus RNA-seq with DroNc-seq. Nature Methods 14, 955-958 (2017).

53. Mu, Q. et al. MYC amplification at diagnosis drives therapy-induced hypermutation of recurrent glioma. ResearchSquare (2021).

54. Sanchez-Vega, F. et al. Oncogenic Signaling Pathways in The Cancer Genome Atlas. Cell 173, 321-337.e10 (2018).

55. Wang, J. et al. Clonal evolution of glioblastoma under therapy. Nature Genetics 48, 768-776 (2016).

56. Louis, D. N. et al. The 2021 WHO Classification of Tumors of the Central Nervous System: a summary. Neuro-Oncology 23, (2021).

57. Johnson, B. E. et al. Mutational analysis reveals the origin and therapy-driven evolution of recurrent glioma. Science 343, (2014).

58. Barthel, F. P. et al. Longitudinal molecular trajectories of diffuse glioma in adults. Nature 576, (2019).

59. Venkataramani, V., Tanev, D. I., Kuner, T., Wick, W. \& Winkler, F. Synaptic input to brain tumors: clinical implications. Neuro-oncology 23, 23-33 (2021).

60. Jung, E. et al. Emerging intersections between neuroscience and glioma biology. Nature Neuroscience vol. 22 1951-1960 (2019).

61. Venkatesh, H. S. et al. Electrical and synaptic integration of glioma into neural circuits. Nature 573, 539-545 (2019).

62. Venkataramani, V. et al. Glutamatergic synaptic input to glioma cells drives brain tumour progression. Nature 573, 532-538 (2019).

63. Osswald, M. et al. Brain tumour cells interconnect to a functional and resistant network. Nature 528, 93-98 (2015).

64. Henley, J. M. \& Wilkinson, K. A. Synaptic AMPA receptor composition in development, plasticity and disease. Nature Reviews Neuroscience vol. 17 337350 (2016).

65. Diering, G. H. \& Huganir, R. L. The AMPA Receptor Code of Synaptic Plasticity. Neuron vol. 100 314-329 (2018). 
66. Tirosh, I. et al. Single-cell RNA-seq supports a developmental hierarchy in human oligodendroglioma. Nature 539, 309-313 (2016).

67. Neftel, C. et al. An Integrative Model of Cellular States, Plasticity, and Genetics for Glioblastoma. Cell 178, 835-849.e21 (2019).

68. Henley, J. M. \& Wilkinson, K. A. Synaptic AMPA receptor composition in development, plasticity and disease. Nature Reviews Neuroscience 2016 17:6 $17,337-350$ (2016).

69. Buenrostro, J. D. et al. Single-cell chromatin accessibility reveals principles of regulatory variation. Nature 523, 486-490 (2015).

70. Cao, J. et al. SINGLE-CELL GENOMICS Joint profiling of chromatin accessibility and gene expression in thousands of single cells. Science vol. 361 (2018).

71. Lareau, C. A. et al. Droplet-based combinatorial indexing for massive-scale single-cell chromatin accessibility. Nature Biotechnology 37, 916-924 (2019).

72. Stoeckius, M. et al. Simultaneous epitope and transcriptome measurement in single cells. Nature Methods 14, 865-868 (2017).

73. Corces, M. R. et al. An improved ATAC-seq protocol reduces background and enables interrogation of frozen tissues. Nature Methods 14 , $959-962$ (2017).

74. Matson, K. J. E. et al. Isolation of adult spinal cord nuclei for massively parallel single-nucleus RNA sequencing. Journal of Visualized Experiments 2018, (2018).

75. Gu, W. et al. Depletion of Abundant Sequences by Hybridization (DASH): Using Cas9 to remove unwanted high-abundance species in sequencing libraries and molecular counting applications. Genome Biology 17, (2016).

76. Loi, D. S. C., Yu, L. \& Wu, A. R. Effective ribosomal RNA depletion for single-cell total RNA-seq by scDASH. PeerJ 9, e10717 (2021).

77. Chen, S., Zhou, Y., Chen, Y. \& Gu, J. Fastp: An ultra-fast all-in-one FASTQ preprocessor. in Bioinformatics vol. 34 (2018).

78. Shen, W., Le, S., Li, Y. \& Hu, F. SeqKit: A cross-platform and ultrafast toolkit for FASTA/Q file manipulation. PLoS ONE 11, (2016).

79. Li, H. seqtk Toolkit for processing sequences in FASTA/Q formats. GitHub 767, (2012).

80. Bushnell, B., Rood, J. \& Singer, E. BBTools Software Package. PLOS ONE vol. 12 e0185056 https://sourceforge.net/projects/bbmap/ (2017).

81. Li, H. \& Durbin, R. Fast and accurate short read alignment with Burrows-Wheeler transform. Bioinformatics 25, (2009).

82. Li, H. et al. The Sequence Alignment/Map format and SAMtools. Bioinformatics 25, (2009).

83. Smith, T., Heger, A. \& Sudbery, I. UMI-tools: Modeling sequencing errors in Unique Molecular Identifiers to improve quantification accuracy. Genome Research 27, (2017).

84. Quinlan, A. R. \& Hall, I. M. BEDTools: A flexible suite of utilities for comparing genomic features. Bioinformatics 26, (2010).

85. Zaccaria, S. \& Raphael, B. J. Characterizing allele- and haplotype-specific copy numbers in single cells with CHISEL. Nature Biotechnology 39, 207-214 (2021).

86. Das, S. et al. Next-generation genotype imputation service and methods. Nature Genetics 48, (2016).

87. Navin, N. et al. Tumour evolution inferred by single-cell sequencing. Nature 472, 90-95 (2011).

88. Nilsen, G. et al. Copynumber: Efficient algorithms for single- and multi-track copy number segmentation. BMC Genomics 13, (2012).

89. Talevich, E., Shain, A. H., Botton, T. \& Bastian, B. C. CNVkit: Genome-Wide Copy Number Detection and Visualization from Targeted DNA Sequencing. PLoS Computational Biology 12, (2016).

90. Oesper, L., Mahmoody, A. \& Raphael, B. J. THetA: Inferring intra-tumor heterogeneity from high-throughput DNA sequencing data. Genome Biology 14, (2013).

91. Oesper, L., Satas, G. \& Raphael, B. J. Quantifying tumor heterogeneity in whole-genome and whole-exome sequencing data. Bioinformatics 30, (2014).

92. Paradis, E. \& Schliep, K. Ape 5.0: An environment for modern phylogenetics and evolutionary analyses in R. Bioinformatics 35, (2019).

93. Desper, R. \& Gascuel, O. Fast and accurate phylogeny reconstruction algorithms based on the minimum-evolution principle. in Lecture Notes in Computer Science (including subseries Lecture Notes in Artificial Intelligence and Lecture Notes in Bioinformatics) vol. 2452 (2002).

94. Dobin, A. et al. STAR: Ultrafast universal RNA-seq aligner. Bioinformatics 29, (2013).

95. Bray, N. L., Pimentel, H., Melsted, P. \& Pachter, L. Near-optimal probabilistic RNA-seq quantification. Nature Biotechnology 34, (2016). 
96. Patro, R., Duggal, G., Love, M. I., Irizarry, R. A. \& Kingsford, C. Salmon provides fast and bias-aware quantification of transcript expression. Nature Methods 14, (2017).

97. Melsted, P. et al. Modular, efficient and constant-memory single-cell RNA-seq preprocessing. Nature Biotechnology 39, (2021).

98. Butler, A., Hoffman, P., Smibert, P., Papalexi, E. \& Satija, R. Integrating single-cell transcriptomic data across different conditions, technologies, and species. Nature Biotechnology 36, (2018).

99. Stuart, T. et al. Comprehensive Integration of Single-Cell Data. Cel/ 177, 1888-1902.e21 (2019).

100. Finak, G. et al. MAST: A flexible statistical framework for assessing transcriptional changes and characterizing heterogeneity in single-cell RNA sequencing data. Genome Biology 16, (2015).

101. Jin, S. et al. Inference and analysis of cell-cell communication using CellChat. Nature Communications 2021 12:1 12, 1-20 (2021).

102. Gómez-Rubio, V. ggplot2 - Elegant Graphics for Data Analysis (2nd Edition) . Journal of Statistical Software 77, (2017).

103. Kassambara, A. ggpubr: "ggplot2" Based Publication Ready Plots. R package version 0.4.0 (2020).

104. Yu, G., Smith, D. K., Zhu, H., Guan, Y. \& Lam, T. T. Y. ggtree: an r package for visualization and annotation of phylogenetic trees with their covariates and other associated data. Methods in Ecology and Evolution 8, (2017).

105. Gu, Z., Eils, R. \& Schlesner, M. Complex heatmaps reveal patterns and correlations in multidimensional genomic data. Bioinformatics 32, (2016).

106. Inkscape.org. Draw Freely | Inkscape. inkscape.org (2020).

\section{Supplementary Figure Legends}

\section{Supplementary Fig. 1 | scONEseq benchmarking.}

a, Lorenz curve of single-cell scWGS data from DR-seq (SK-BR-3 cells), G\&T-seq (mouse embryo 8-cells stage cells), and scONE-seq (frozen tumor nuclei) in a representative normal chromosome region (DR-seq and scONE-seq: human chr2; G\&T-seq: mouse chr1 and chr19 chosen for comparable length to human chr2). Percentiles of the genome covered are plot against the cumulative fraction of reads. A perfect coverage uniformity results in a straight line with slope 1. Variations within each method are plotted using \pm SD. scONE-seq is closest to the bulk control, indicating better uniformity for copy number calculation.

b, Comparison of single-cell scWGS data dispersion from DR-seq (SK-BR-3 cells), G\&T-seq (mouse embryo 8-cells stage cells), and scONE-seq (frozen tumor nuclei) in a representative normal chromosome region (DR-seq and scONE-seq: human chr2; G\&T-seq: mouse chr1 and chr19 chosen for comparable length to human chr2). The box plot shows a significant technical improvement of scONE-seq over the other two methods, even though scONE-seq has lower average DNA sequencing depth in this comparison.

c, Scatter plots show the correlation between detected quantity of ERCC spike-in and original input concentration of each ERCC molecule, comparing Smartseq2 $(1 \mu \mathrm{l}$ 1:500,000 ERCC, $n=3)$, scONE-seq $(1 \mu \mathrm{l} \mathrm{1:500,000} \mathrm{ERCC,} n=3)$, DR-seq $(0.2 \mu \mathrm{l}$ 1:500,000 ERCC, $n=21)$, and G\&T-seq $(2 \mu \mathrm{l} 1: 500,000$ ERCC, $n=32)$. Of the methods compared, only scONE-seq shows comparable sensitivity and accuracy as Smart-seq2, an RNA-only amplification method.

c, Gene detection sensitivity for mock scONE-seq RNA ( $n=3)$, mock scONE-seq DNA+RNA $(n=3)$, and Smart-seq2 $(n=3)$. Downsampled to similar sequencing depth ( 0.15 million mapped reads) $(P=0.0019$, ANOVA test). Shown are numbers of genes detected over 1 count.

d, Precision evaluation with HCT116 cells. The most commonly expressed 8000 genes were used for scONE-seq $(n=86)$ and Smart-seq2 $(n=94)$ respectively. The box plots show the pairwise coefficient of determination $\left(R^{2}\right)$ values of cells with Pearson correlations from log-transformed TPM values.

e, Estimated saturation plot with mock sample expression data from scONE-seq RNA, scONEseq DNA+RNA, and Smart-seq2.

f, Mapped region statistic. The difference between HCT116 DNA+RNA group (RNA) mapped regions and HCT116 RNA alone control is $2.58 \%$.

\section{Supplementary Fig. 2 | Markers used in cell-types annotation.}

a, Dot plot of common markers for 4 cell lines.

b, Scatter plots to show important markers used in cell-types annotations with the scONE-seq dataset (top) and Smart-seq2 dataset (bottom).

c, Scatter plots to show 3 markers for identifying the existence of yठ T cells with the scONE-seq dataset (top) and Smart-seq2 dataset (bottom).

d, scONE-seq captured $\mathrm{T}_{\text {reg }}$ cells (FOXP3+, CCR4t);

e, Non-polyA genes like PZP and SESN3 are captured with scONE-seq data.

Supplementary Fig. 3 | Copy number profiles of 3 NPC43 clones. 
a, b, c, d, Dots plot with normalized counts across the human genome and solid line plots for corresponding estimated integer copy number. Amplification regions are highlighted with red; deletion regions are highlighted with light blue. Data from the NPC43 cell line establishment state (WGS data, a), the NPC43 C1 clone pseudo bulk data ( $b, n=20)$, the NPC43 C2 clone pseudo bulk data $(\mathbf{c}, n=19)$, and the NPC43 C3 clone pseudo-bulk data (d, $n=69)$.

e, Annotate the clonal information to UMAP with transcriptome data; copy number changes do not impact the gene expression profiles significantly in this case.

\section{Supplementary Fig. 4 | Clinic histological photomicrographs.}

a, Representative H\&E (first row, 200X) and immunohistochemical stains (2-4 rows, 400X) of the primary tumor, $1^{\text {st }}$ recurrence, and $2^{\text {nd }}$ recurrence of the IDH1mutant astrocytoma. The primary and recurrent tumors demonstrate similar histologic characteristics featured by pleomorphic hyperchromatic astrocytic cells with brisk mitosis (arrow) (H\&E), endothelial proliferation (arrowhead) (H\&E), and necrosis (asterisk) (H\&E, $2^{\text {nd }}$ recurrence). The tumors show cytoplasmic positivity for IDH1-R132H (second row), loss of ATRX expression (third row), and nuclear positivity for p53 (fourth row).

\section{Supplementary Fig. 5 | The genome information of the 2R IDH1-mutant astrocytoma.}

a, Heatmap showing the normalized counts of the DNA data from scONE-seq profiling of the 2R IDH1-mutant astrocytoma. 4 genome states, normal cell ( $n=$ $586), 2 R$ clone $1(n=17), 2 R$ clone $2(n=20), 2 R$ clone $3(n=432)$, are presented to show the CNAs in different cells.

b, The FACS sorting plot showing the DAPI intensity density of nuclei from the frozen tumor sample. Most of the cells are diploid. The doubled or even higher DAPI intensity indicates the aneuploid cells with presumed genome duplication.

c, Heatmap showing the BAF imputed from scONE-seq DNA data. 4 genome states, normal cell $(n=586), 2 R$ clone 1 ( $n=17), 2 R$ clone $2(n=20), 2 R$ clone 3 ( $n$ = 432), are presented to show the BAF in different cells.

d, Heatmap showing the mirrored BAF imputed from scONE-seq DNA data. 4 genome states, normal cell $(n=586), 2 R$ clone $1(n=17), 2 R$ clone $2(n=20), 2 R$ clone $3(n=432)$, are presented to show the LOH in different cells.

e, Heatmap showing CNAs from WES and scONE-seq clonal pseudo-bulks. The 2R clone 1 and WES inferred P clone 1 both have chromosome $4 \mathrm{q}$ deletion.

f, Dots plots showing the chromosome 9 genome states in 3 clones. The CDKN2A homozygous deletion happened in 2 R clone 2 and clone 3.

\section{Supplementary Fig. 6 | Characterization of 2R clone 1 cells.}

a, Scatter plots show the expression pattern of 4 key markers to distinguish the $2 \mathrm{R}$ clone 1 cells in the scONE-seq RNA dataset. XIST (deletion in clone 3 ), RFX3 (homozygous deletion in clone 3), ADCY8 and GRIA1 (unique expression in clone 1 compared to normal astrocytes).

b, UMAP of $10 X$ Genomics snRNA-seq data validating the cell type composition found using scONE-seq.

c, Dot plot with 10X Genomics snRNA-seq shows markers used to annotate cell types.

d, Scatter plots show the expression pattern of 4 key markers to distinguish 2R clone 1 cells in the 10X Genomics snRNA-seq dataset. XIST (deletion in clone 3), RFX3 (homozygous deletion in clone 3), ADCY8 and GRIA1 (unique expression in clone 1 compared to normal astrocytes).

e, Violin plots show the expression pattern of the markers selected for immunostaining validation, IDH1 and $A D C Y 8$.

\section{Supplementary Fig. 7 | Analysis of 2R clone 1 distribution and gene expression in the context of the tumor microenvironment.}

a, Tumor section fluorescence images showing the spatial distribution of the IDH1 (R132H) and ADCY8 positive cells in the FFPE sections (both primary and 2R tumors). Regions with strong ADCY8 signals were circled with the green line. These regions appear to be 'normal-adjacent' tissues (low IDH1 (R132H) signals indicated with yellow arrows).

b, Scatter plots showing the expression pattern of astrocytic gene APOE and AMPAR subunit GRIA1 co-expression in many 2 R clone 1 cells.

c, Heatmaps shows the potential communication strength between cell types in different secretory pathways. Each row indicates a certain secretory pathway. Left shows the potential to send out certain pathway ligands; Right heatmap shows the potential to receive certain pathway signals with receptors expression. 2R clone 1 cells were predicted to be an important source of the TGF $\beta$ ligand source.

\section{Figures}


Fig. 1

a
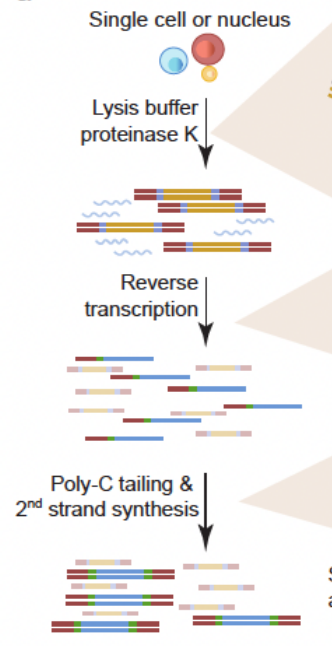

e

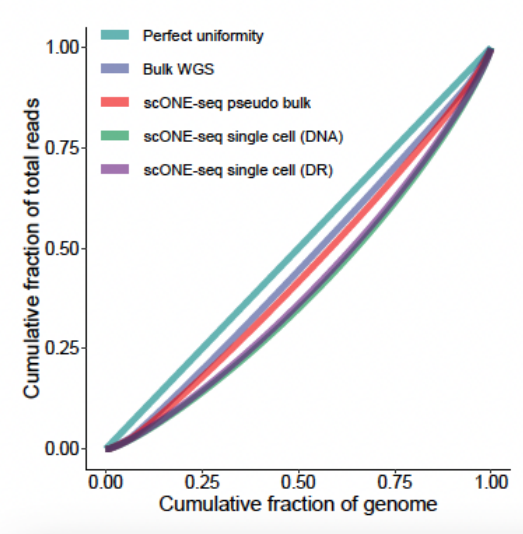

b

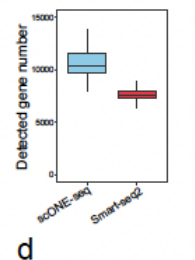

Annealing sequence

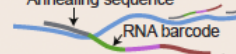

Amplification primer

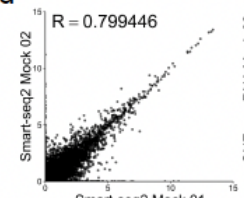

Smart-seq2 Mock 01

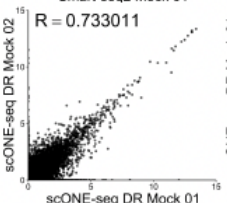
cDNA and gDNA library c
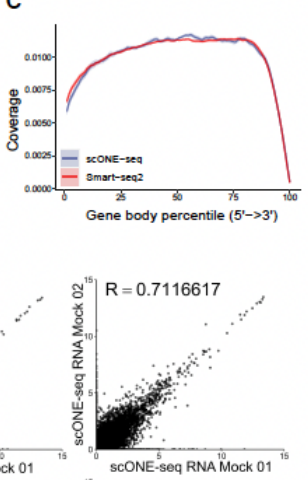

$5^{15} \mid \mathrm{R}=0.7205361$

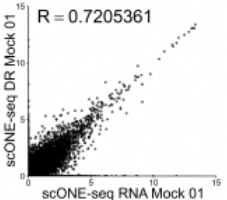

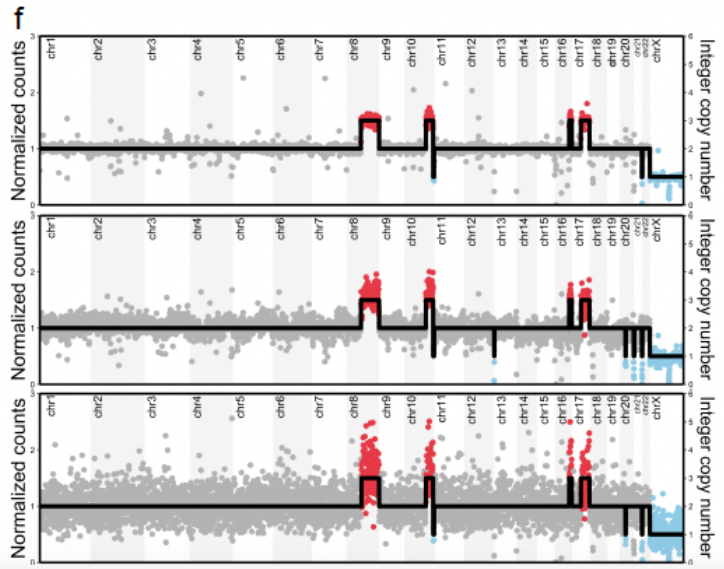

\section{Figure 1}

Overview of scONE-seq: schematic and benchmark. a, Workflow of scONE-seq. DNA and RNA barcodes are added by Tn5 with custom adaptor and RT primers respectively. P7-Illumina sequencing primer is included in this pre-amplification process. P5-Illumina sequencing primer is added in the later library

construction step with a second Tn5 tagmentation custom adaptor. b, Gene detection sensitivity for scONE-seq ( $n=86$, HCT116 cells) and Smart-seq2 ( $n=94$, HCT116 cells), downsampled to similar sequencing depth ( 0.2 million mapped reads) ( $<<2 \times 10-16$, t-test). Shown are numbers of genes detected over 1 count. c, Gene body coverage for scONE-seq $(n=86)$ and Smart-seq2 $(n=94)$. Cell-cell variations are shown with error areas. $d$, Accuracy across mock samples for scONE-seq (RNA control, DNA+RNA group) and Smart-seq2. Pearson correlations were calculated from log-transformed TPM values. e, The Lorenz curve of bulk and scONE-seq single-cell data. Percentiles of the genome covered are plot against the cumulative fraction of reads. A perfect coverage uniformity results in a straight line with slope 1. f, g, h, Dots plot with normalized counts across the genome and solid line plots for corresponding estimated integer copy number. Amplification regions are highlighted with red; deletion regions are highlighted with light blue. Data from the bulk HCT116 whole genome sequencing (e), the HCT116 scONE-seq pseudo-bulk data ( $\mathrm{f}, \mathrm{n}=86$ ), and a single-cell HCT116 scONE-seq data (g). 
Fig. 2
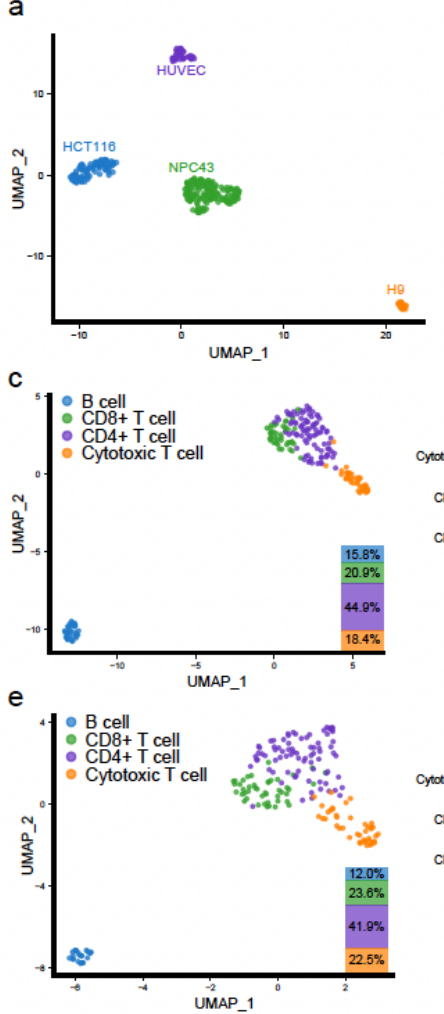

g

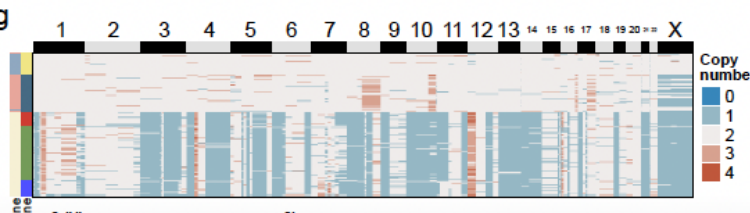

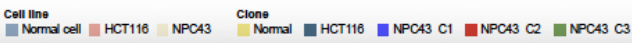

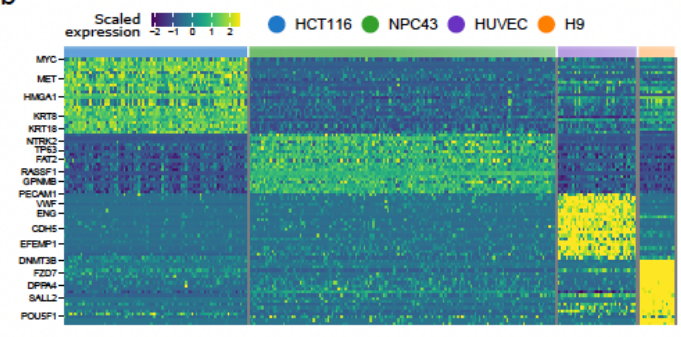

d

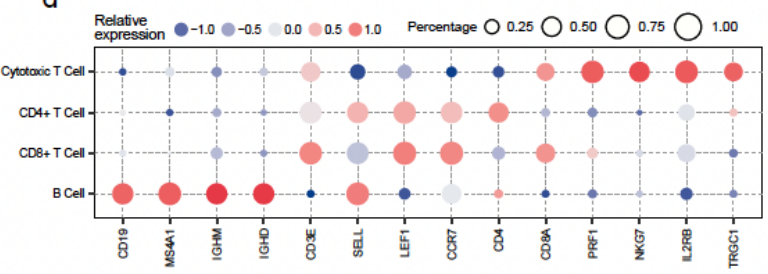

f

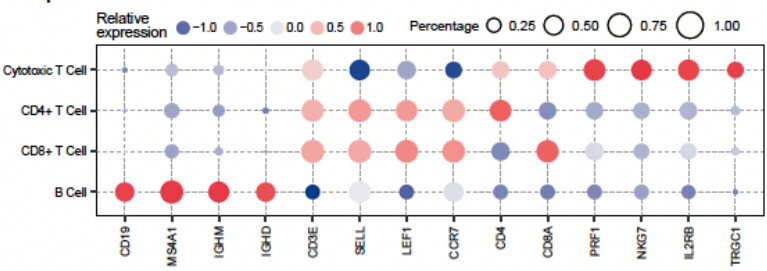

h

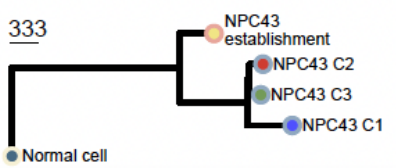

Figure 2

scONE-seq cell-types classification and CNAs clone identification. a, UMAP of scONE-seq cell lines RNA data, cells from same the cell line are clustered together. b, Differential expression genes (DEGs) heatmap; DEGs separate the cells based on their cell type; common markers for these cell lines are labeled in the heatmap. c, e, UMAP of lymphocytes RNA data from scONE-seq (c) and Smart-seq2 (e); cell-types annotations are based on known markers of immune cells. Cell-type composition shows no difference between two datasets $(P=0.5109$, Chi-squared test). $d$, $f$, Dot plots of markers used for cell-types annotations with the scONE-seq dataset (d) and Smart-seq2 dataset (f). g, Copy number profiles calculated with scONE-seq cell line DNA dataset; cells are organized by hierarchical clustering (normal $n=27 ;$ HCT116 $n=48$; NPC43 $n=108$ ). h, The minimum evolution tree with diploid as root; NPC43 cells used in this study acquired more CNAs compared with the genome state when the cell line was established. Unit shows the Manhattan distance. 
Fig. 3

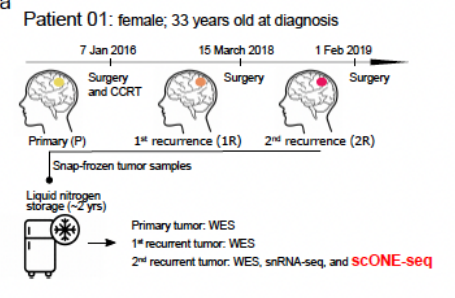

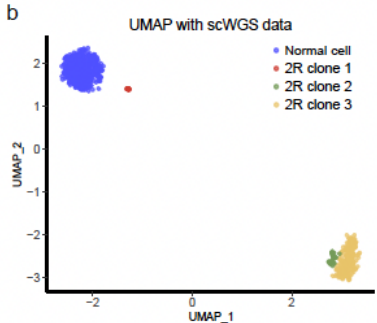

d d

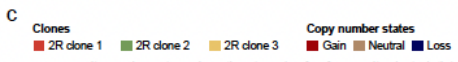

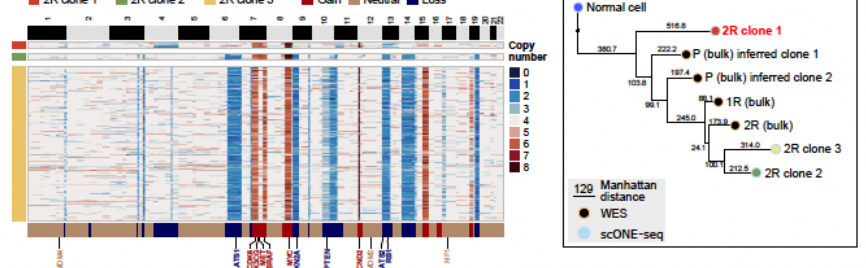

e
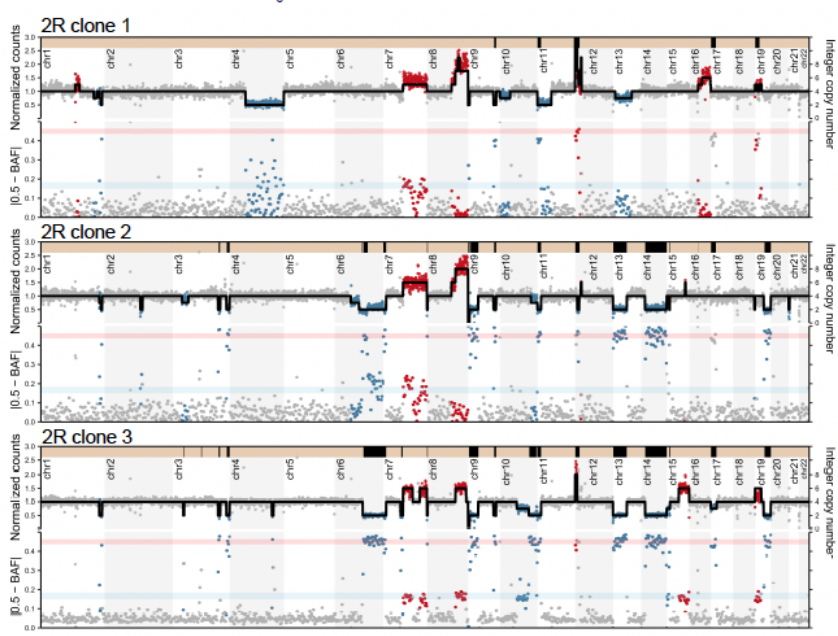

Figure 3

scONE-seq reveals the clonal composition of the IDH1-mutant astrocytoma. a, Schematic showing the patient history for the sample used in this study. The patient has been diagnosed as IDH1-mutant (Grade IV) astrocytoma. Surgery was performed to excise the tumor, and concurrent chemoradiotherapy (CCRT) was then applied. The recurrences of the tumor were excised without further drug treatment. Tumor specimens were snap-frozen in the liquid nitrogen tank and stored for two years before subject to nuclei extraction. b, UMAP of scONE-seq DNA copy number data shows the 4 genome states in this IDH1-mutant astrocytoma sample. $2 \mathrm{R}$ - second recurrence. c, Heatmap of integer copy numbers of all the $2 \mathrm{R}$ astrocytoma cells profiled; 3 clones with different copy number profiles are observed. The bottom annotation bar represents the CNAs in this tumor sample. Some commonly known glioma/astrocytoma driver genes are shown. Amplified genes are highlighted with red; deleted genes are highlighted with dark blue. d, The minimum evolution tree with diploid as root; the WES data inferred CNV from the same patient were integrated to show the evolutionary relationship between the tumor recurrences and their various clones. The P clones are inferred from bulk WES data (see Methods). P - primary; $1 \mathrm{R}$ - first recurrence. e, The top panel shows the dots plot with normalized counts across the human genome and the solid line representing the estimated integer copy number. The bottom panel shows the mirrored BAF dots plot across the genome. Relatively amplified regions are highlighted with red; relatively deleted regions are highlighted with blue. If dots are close to the red belt in the mirrored BAF dots plot, this indicates that there are $\mathrm{LOH}$ in those regions. If dots are close to the blue belt in the mirrored BAF dots plot, this indicates that there are imbalanced haplotypes in that regions. The top bar highlights the $\mathrm{LOH}$ regions of the genome. The clonal pseudo-bulk genome information for each $2 \mathrm{R}$ clone is also shown. 
Fig. 4

a

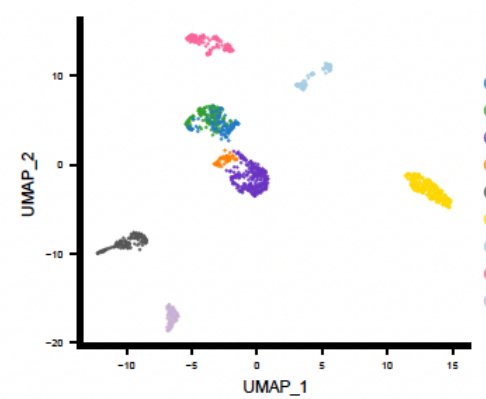

C

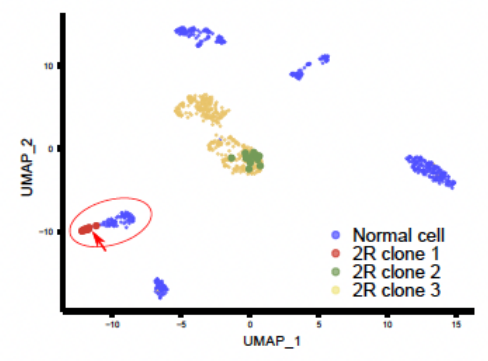

b

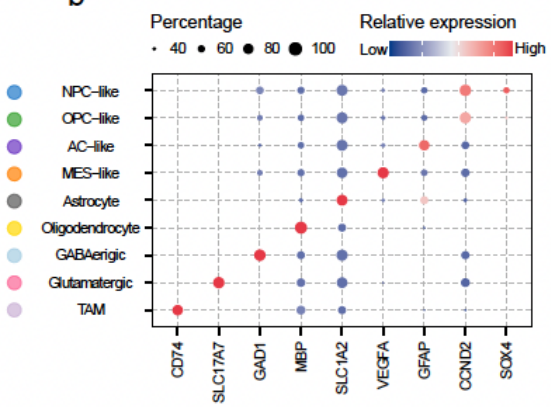

d
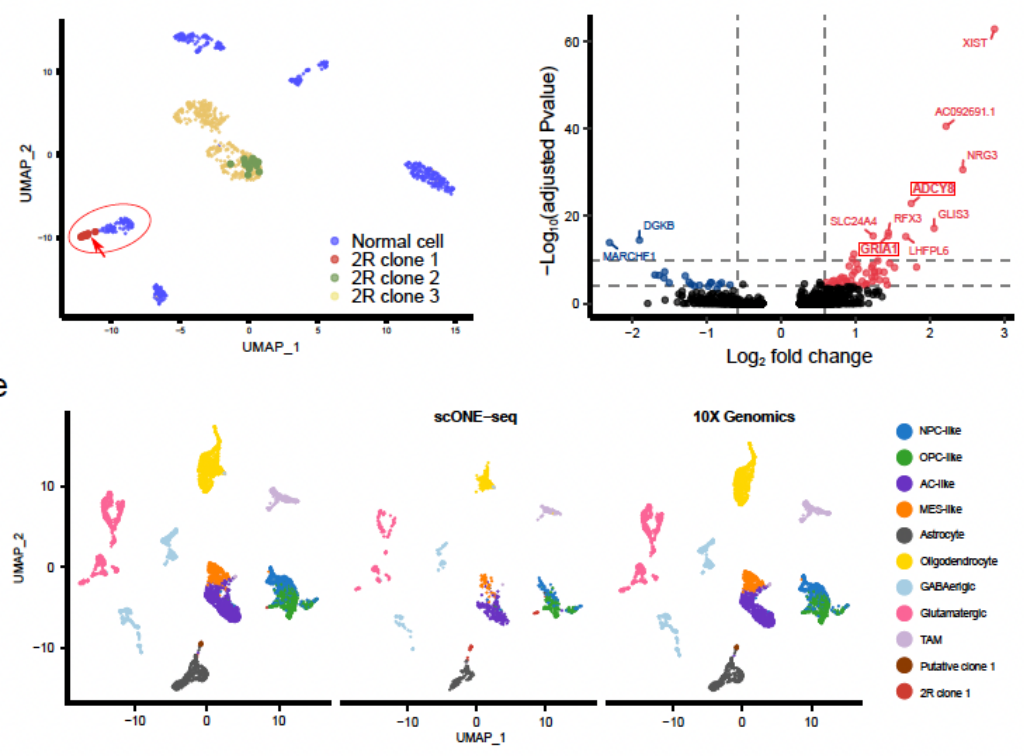

Figure 4

scONE-seq reveals the tumor microenvironment composition of an IDH1-mutant astrocytoma. a, UMAP of scONE-seq RNA data shows the TME composition cell types in this second recurrence IDH1-mutant astrocytoma sample. Tumor cells are classified into 4 cellular states based on their meta-module scores. b, Dot plot shows some markers for the annotation of cell types. c, UMAP of scONE-seq RNA data annotated with clonal information. The $2 \mathrm{R}$ clone 1 cells are clustered with normal astrocytes. $d$, Volcano plot shows the DEGs between the $2 \mathrm{R}$ clone 1 and clone 3 cells. Genes with higher expression in clone 1 cells are colored with red. Genes with higher expression in clone 3 are colored with blue. e, UMAP showing the integration of scONE-seq and 10x snRNA-seq dataset. The integrated data (left) retains all cell types identified with scONE-seq or 10x snRNA-seq. The split-UMAP (right) shows adequate mixing of cell-types found separately by the 2 methods, with clone 1 cells (from scONE-seq) and putative clone 1 cells (from snRNA-seq) falling into the same integrated cluster. 
Fig. 5

a

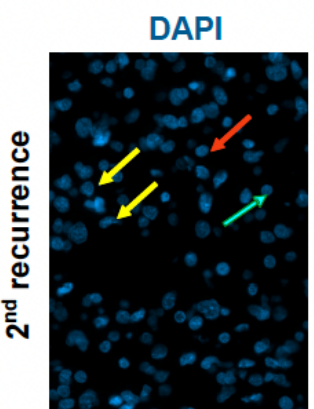

IDH1(R132H)

ADCY8

Merge
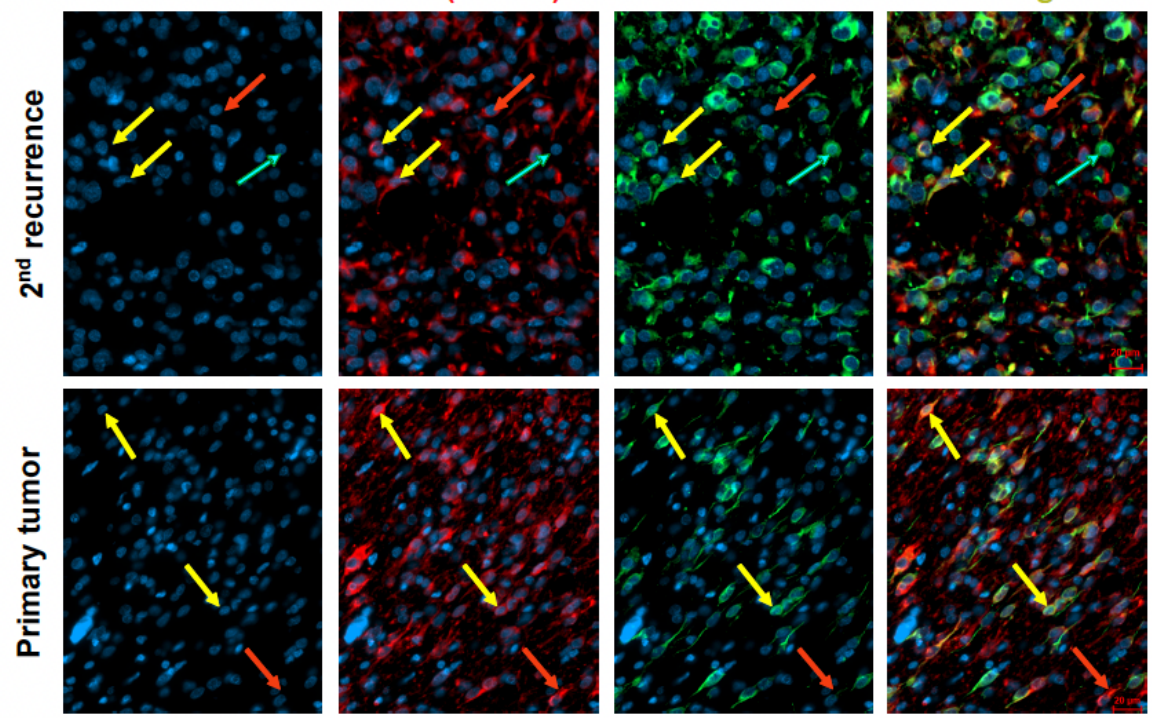

b

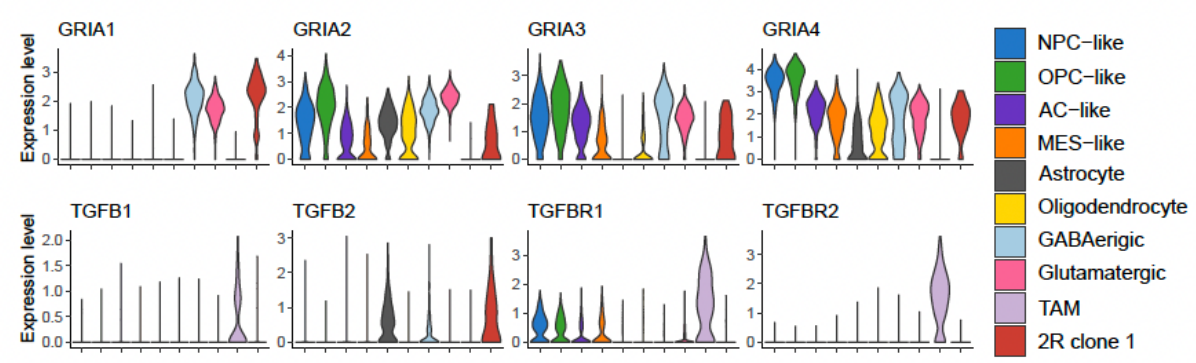

\section{Figure 5}

Characterization of $2 \mathrm{R}$ clone 1 features. a, The immunofluorescent images co-label the IDH1 (R132H) and ADCY8 in the FFPE section of the patient. The top panel shows images from the $2 R$ tumor; the bottom shows images from the primary tumor from the same patient. The yellow arrow indicates the co-stained putative clone 1 cells; the red arrow indicates the other tumor cells; the Green arrow indicates normal astrocytes or GABAergic neurons. b, The AMPAR subunitencoding gene expression pattern in this 2R IDH1-mutant astrocytoma. GRIA1 is highly expressed in clone 1 cells. c, The TGF $\beta$ signaling genes expression pattern in this $2 \mathrm{R}$ IDH1-mutant astrocytoma. TGFB2 is highly expressed in clone 1 cells and normal astrocytes. The receptors are mostly expressed in TAMs.

\section{Supplementary Files}

This is a list of supplementary files associated with this preprint. Click to download.

- 20211013supplementarynote.docx

- 20211013SFigsall.pdf

- SupplementaryTable1.xlsx

- SupplementaryTable2.xlsx 